\title{
Towards 3D integral-imaging broadcast with increased viewing angle and parallax
}

\author{
A. Dorado ${ }^{1,}{ }^{*}$, S. Hong $^{1}$, G. SaAvedra ${ }^{1}$, J.C. Barreiro ${ }^{1}$, B. JAVidi ${ }^{2}$, M. Martinez-Corral ${ }^{1}$ \\ ${ }^{1}$ Optics department, University of Valencia, Burjassot, E46100, Spain \\ ${ }^{2}$ Electrical and Computer Engineering Department, University of Connecticut, Storrs, CT 06269 USA. \\ *Corresponding author: adrian.dorado@uv.es
}

We propose a new method for improving the observer experience when using an integral monitor. Our method permits to increase the viewing angle of the integral monitor, and also the maximum parallax that can be displayed. Additionally, it is possible to decide which parts of the 3D scene are displayed in front or behind the monitor. Our method is based, first, in the direct capture, with significant excess of parallax, of elemental images of 3D real scenes. From them, a collection of microimages adapted to the observer lateral and depth position is calculated. Finally, an eye-tracking system permits to determine the 3D observer position, and therefore to display the adequate microimages set. Summarizing, it is reported here, for the first time we believe, the application of eye-tracking technology to the display of integral images of 3D real scenes with bright background. Although we are reporting here only a proof-of-concept experiment, this result could have direct application in a close future for the broadcasting of 3D videos recorded in professional studio, for videoconferences or for on-line professional meetings.

OCIS codes: (120.2040) Displays; (100.4999) Target tracking; (100.6890) Three-dimensional image processing; (110.6880) Three-dimensional image acquisition; (110.2990) Image formation theory.

\section{INTRODUCTION}

Integral imaging (Inl) is a technique that allows the capture and display of 3D information from 3D scenes, either computer generated or from the real world. Inl has been an important research topic in the recent years, mainly due to its capacity to display autostereoscopic 3D images with full parallax and to capture the 4D lightfield of a 3D scene [1-7]. The latter has many useful applications such as obtaining depth maps, performing digital refocusing, removing occlusions, making object recognition, and many others [3-7].

Nowadays, 3D display still remains as one of the most exciting applications of Inl. The core technology is still being refined, and there have been attempts to improve Inl by combining it with other technologies. This is the case of eye-tracking Inl, where the position of the observer is tracked in order to offer her/him a tuned and better experience. The first approach to use this hybrid method was done by G. Park et al. [8] and [9], who determined the observer position with the help of an infrared (IR) camera and two IR LEDs equipped in a goggle. By using the tracking information, the displayed microimages were adapted to the observer position. However, in this research the technique was demonstrated only for computergenerated 3D scenes that were composed only by two planar objects over a black background. Another approach was followed by S. Hong et al. [10], who used a Kinect device to track the observer position. Again in this research the displayed 3D scenes were generated by computer and composed by only two planar objects. Later, $X$. Shen et al. [11] determined the observer position with the help of an IR camera and a LED track clip. Also in this approach, the technique was demonstrated only for computer-generated 3D scenes composed only by two 2D objects over a black background. The main advance was that the displayed microimages were adapted not only to the observer position, but also to potential rotations of the 
observer head. Finally, Z.L. Xiong et al. [12] followed a different approach, since for the computation of the microimages they used an array of virtual orthographic cameras with the same point of convergence. The main lacks of this technique are that orthographic cameras are unrealistic from the optics point of view, and that the method was demonstrated only for synthetic scenes over a black background.

In this paper, we propose a new method to increase the viewing angle of an integral monitor using an eye-tracking system and the SPOC 2.0 algorithm [13]. In addition, we increase the parallax that the integral monitor can display. We also allow the observer to choose which parts of the displayed 3D image are reconstructed in front or behind the lens array of the integral monitor. These improvements are done by dynamic modification of the information displayed on the pixels of the integral monitor depending on the observer 3D position. Our proposal is based in the use of a Kinect device for the tracking system, in the use of SPOC 2.0 for the selection of the reference lane, and in the smart cropping of the microimages according to the 3D location of the observer. The main contributions of the present the work are: first, this work deals for the first time with real scenes and with a fixed number of real cameras. Working with real optics and with real scenes (with real background) is not a minor challenge. Note that in computer-generated images, there is no limitation in the number and positions of the cameras, there are not aberrations, and the crosstalk is strongly reduced since in that case the background can be eliminated. Second, our approach permits, for the first time, to adapt the reference plane of the displayed image to the depth position of the observer.

\section{INTEGRAL IMAGING MONITOR}

In Inl, the display of 3D images is performed via the so-called integral monitor. Integral monitors do not need the use of any type of special glasses, and have the advantage of displaying 3D images with full parallax. But the most important feature is that they reproduce the light distribution of the original 3D scene. Since the observer can adjust her/his accommodation and visual-axes convergence to that light distribution, the convergence-accommodation conflict is avoided [14, 15]. In order to set up an integral monitor, an array of microlenses (MLA) is placed in front of a pixelated display in such a way that the pixels are just at the focal plane of the MLA, and therefore their images are formed at the infinite. In the conventional realization of an integral monitor, just behind each microlens it is displayed a microimage so that both the microlens and the microimage have the same size. The microimages can be obtained directly with a plenoptic camera or transformed from the elemental images captured with an array of digital cameras [13, 16-19].

Once the microimages are displayed on the pixelated display, the microlenses integrate the light rays emitted by the pixels, forming a luminous 3D image, see Fig. 1. As these light rays carry the lightfield information of the 3D scene, its radiance distribution is replicated in the neighborhood of the MLA. The plane of the displayed 3D scene that coincides with the MLA plane is named here as the reference plane $[7,13]$. The reference plane is important because it determines which parts of the 3D scene are displayed in front or behind the monitor. The spatial resolution of displayed images is determined by the MLA pitch. The angular resolution (angular distance between displayed perspectives) depends on the number of pixels per microimage. In other words, corresponding pixels of adjacent microimages contain different perspectives of the same point of the scene and each microimage store information of different points of the 3D scene.

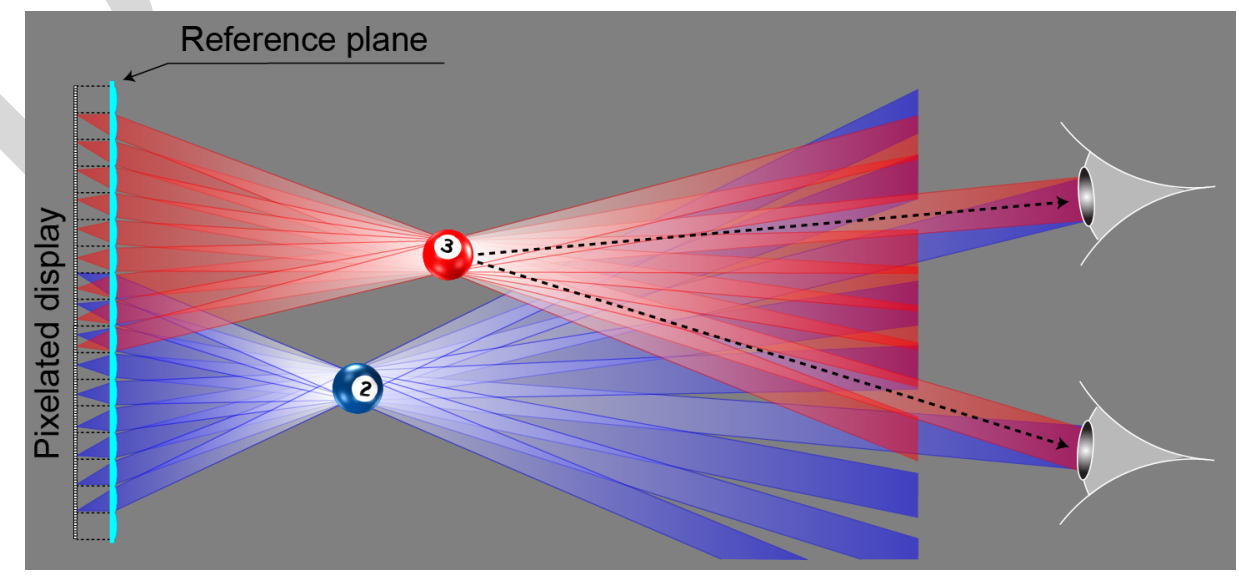


Fig. 1.- The 3D image is reconstructed in the neighborhood of the lens array plane. The eye only receives light from one pixel of each microimage. The crosstalk effect appears when the observer sees rays coming from wrong lenses.

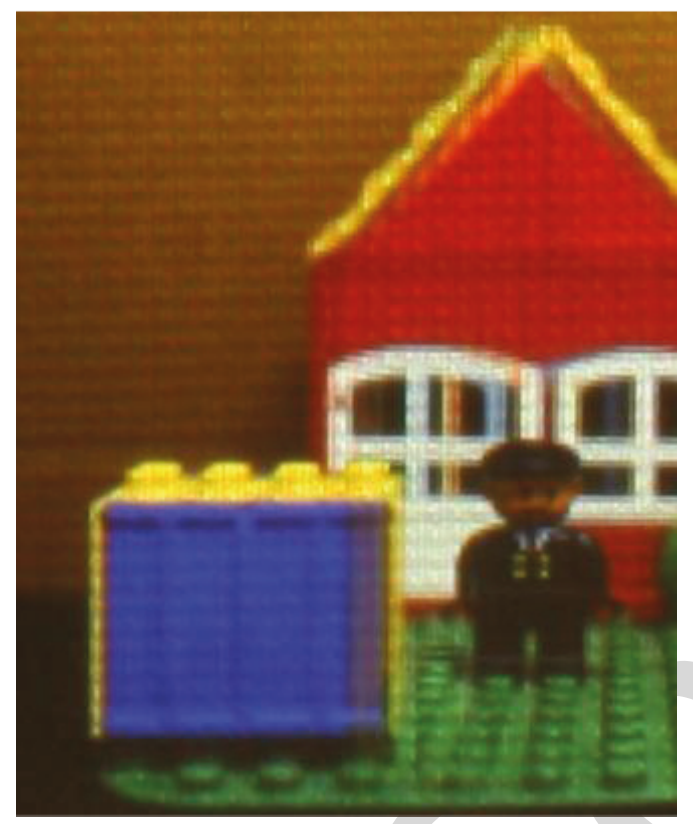

Fig. 2.- The crosstalk causes a distortion on the image perceived by the observer. On this image, the crosstalk can be seen on the roof and the door of the house and, also, on the right side of the block situated at the front of the scene.

It is worth noting that an observer usually only sees a limited bundle of the rays coming from the integral monitor. Due to several factors (observation distance, pixel and lens size, distance between the lenses and the pixels, etc.), only rays proceeding from one pixel are seen by the observer through each microlens, see Fig. 1 . On the other hand, the quality of the perceived 3D image depends on the position of the observer. An integral monitor has a limited area in front of it where the observer can be located in order to perceive a good 3D image. We call this zone as the integral-monitor viewing area. If the two eyes of the observer are inside the viewing area she/he perceives the 3D image with full parallax. If the observer goes outside the viewing area, she/he will see a distorted 3D image. The reason is that some of the light rays emitted by the integral monitor are received by the observer from the wrong lenses, causing a crosstalk effect, see

Fig. 2. To obtain the viewing area of an integral monitor, the positions in which the observer receives the correct information from all the lenses should be found. This is made by projecting each microimage through the center of its lens and into the 3D space in front of it. The area in common for all the projections defines the viewing area $(V A)$ of the integral monitor, see Fig. 3. At a specific depth plane, the projection of a microimage $\left(M I_{\text {Proj }}\right)$ and the viewing area are calculated as

$$
\begin{gathered}
M I_{\text {Proj }}=\frac{p \cdot z}{g} \\
V A(z)=\frac{p \cdot z}{g}-(n-1) p-\mu
\end{gathered}
$$


In these equations $g$ is the gap between the pixels and the lens array, $p$ is the pitch, $n$ is the number of microimages, $\mu$ is the inter-pupillary distance, and $z$ is the projection distance as measured from the MLA. It is easy to see from the equations and from Fig. 3 that the number of microimages of the integral monitor limits the viewing area. Therefore, the higher the number of microimages the smaller the viewing area.

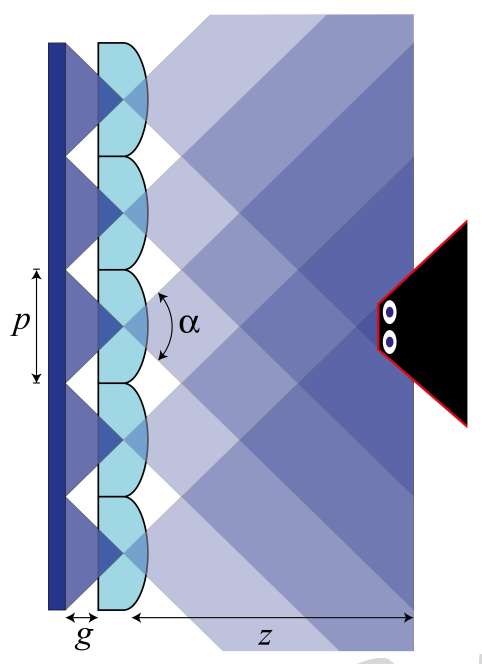

Fig. 3.- Projections of the microimages to a distance $z$. The viewing area is highlighted.

Other parameter of great interest is the viewing angle of the integral monitor. The viewing angle is a monocular parameter, and is defined as the angle formed by the normal to the central microlens and the line that connects the center of the entrance pupil of the eye and the optical center of the central microlens. Since the observer's eye must be placed inside the viewing area, the viewing angle is usually small.

\section{METHOD}

The first step of our proposal is the capture of multi-perspective information of the 3D scene. Such information must be captured with significant excess of parallax. In such case, the resulting microimages are bigger than the corresponding microlenses, and therefore need to be cropped. The eccentricity of the cropping area is determined by the lateral position of the observer. Having an excess of perspective content, one can select which views will compose the cropped microimages and, therefore, control which ones will be displayed and seen by the observer. The cropping process is then an important part of our method for increasing the maximum parallax displayable by the integral monitor. The second step consists of dynamically changing the displayed information to improve the viewing experience. This is done by modifying the displayed microimages depending on the observer position. A kinect-based eye-tracking system provides the control software with the 3D position of the observer in real time. With that information, the control software can modify the displayed microimages dynamically. Specifically, the software shifts the microimages being displayed on the integral monitor, changes their perspectives, and changes the reference plane. Next, we detail the process.

\subsection{Shifting the cropping masks}

In the data base of the computer we have a collection of microimages, each significantly bigger than the corresponding microlenses. In order to compose the full integral frame, each microimage must be cropped. The size of the cropping mask is equal to the MLA pitch. When the observer is placed just in front of the central microlens, the cropping masks are set at the center of the microimages, and the cropped microimages are just in front of the corresponding lenslets. However when the observer moves laterally the cropping masks are shifted proportionally. Additionally, to compose the integral frame the cropped microimages are shifted in the same proportion so the viewing area follows the movement of the observer, see Fig. 4. 


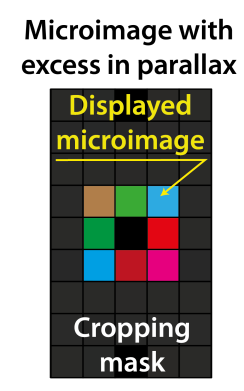

(a)

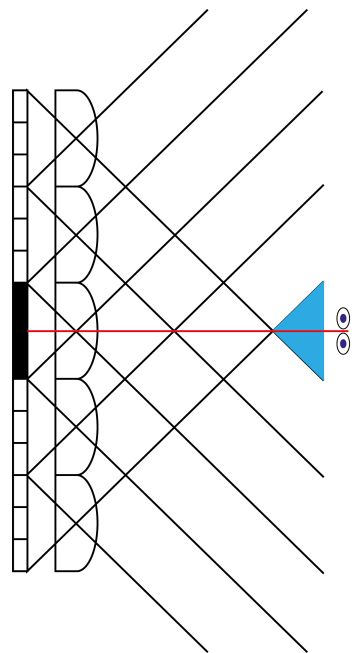

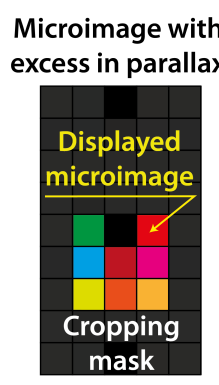

(b)

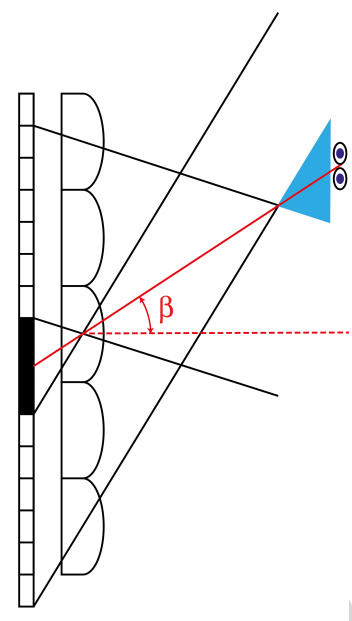

Fig. 4 (a) When the observer is just in front of the central microimage, the cropping masks are placed at the center; (b) The cropping masks and microimages are shifted to allow the viewing area to match the observer's movement. The viewing angle, $\beta$, increases significantly as compared with the conventional configuration.

Note that because an observer only sees one pixel through each microlens, this shift happens when the observer goes to see an adjacent pixel. This distance can be calculated as $\Delta(z)=z \delta / g$, where $\delta$ is the pixel size. Therefore, we can consider the 3D space in front of the integral monitor divided into sectors, each one corresponding to the projection of a pixel of the integral monitor into the real 3D space, see Fig. 5. In this consideration, when the observer moves from one sector to another, the control software makes the shifts.

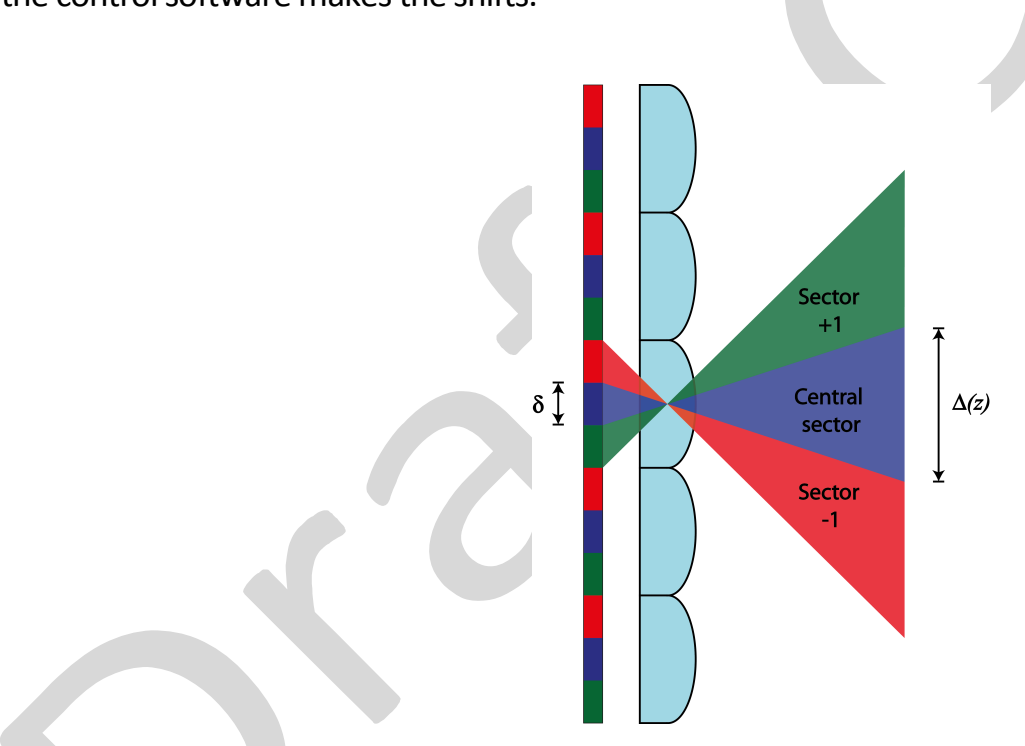

Fig. 5.- The 3D space in front of the integral monitor is divided into sectors, each corresponding to the projection of a pixel through the central microlens. When the observer moves from one sector to the neighbor one, the control software modifies the displayed microimages.

Moreover, in our approach the observer must be always at the center of the viewing area. Therefore, the cropping mask of the microimages needs to be aligned with the observer and the center of its corresponding lenses. So, if the observer is just in front of the integral monitor, on the central sector, the microimages being displayed are not shifted, as in a conventional integral monitor, see Fig. 4 (a). However, if the observer is at any other sector, the cropping mask will be shifted a number of pixels equal to the number of sectors between the central sector and that one (inclusive), see Fig. 4 (b). Note that the microimages are always displaced towards the opposite direction the observer has moved.

\subsection{Changing the reference plane}


Finally, to further improve the visualization experience, our method allows the observer to change the reference plane at will. To that end, the control software changes the reference plane of the displayed microimages depending on the distance between the observer and the integral monitor. The advantage of this proposal is that the observer can control with her/his positioning which parts of the 3D scene will be reconstructed in front or behind the lens array. To change the reference plane of the microimages we use the SPOC 2.0 algorithm [13].

\section{EXPERIMENT}

To illustrate the proposal, we have applied our method to an integral monitor. To make it easier, we decided to increase only the horizontal parallax of the displayed 3D image. The first step is to set up the integral monitor. In previous realizations, we used a tablet as the pixelated display and a small lens array [13, 20-22]. This time we have used a 24 inches, $4 \mathrm{~K}$ monitor (Dell-P2415Q ) with $3840 \times 2160$ pixels. The lens array employed is composed by $327 \times 184$ lenses with pitch $p=1.607 \mathrm{~mm}$ and a focal length $f=8 \mathrm{~mm}$. In order to avoid the facet braiding effect $[23,24]$ it is recommended to place the lens array parallel to the screen, in such a way that the pixels are at the focal plane of the lenses. However, this causes some color moiré effect created by the magnification of the subpixel structure [25]. To avoid this drawback we displaced slightly the lens array to a gap $g=8.4 \mathrm{~mm}$. This new position reduces noticeably this color moiré effect without sacrificing the quality of the displayed image. At this point, a topic of great interest was to estimate the influence of aberrations in the quality of the displayed, and perceived, images. Note that in our display system the matter of interest is not the image of points, but the light emitted by a given pixel, passing through the corresponding microlens, and finally impinging the eye pupil. We did some simulations (not shown here) with the help of commercially available optical-design software (Optalix ${ }^{\circledast}$ ) and found that the influence of off-axis aberration in the quality of observed images is negligible.

As for the capture of the plenoptic field, we propose the synthetic aperture method [26]. The number of pixels per microlens in our integral monitor, $12 \times 12$, is equal to the perspectives our integral monitor can display simultaneously. Then, in order to have excess in the captured parallax, for the posterior cropping, we captured a total of 40x12 elemental images (Els). The camera used to capture the different Els was a Canon 450D, which was mounted on a platform whose position and displacement were controlled by LabVIEW ${ }^{\circledR}$ code. The lateral pitch of the displacement was $P=5 \mathrm{~mm}$ and the chosen 3D scene was composed of a Lego ${ }^{\circledR}$ house, a doll and a box placed at 39, 48 and $55 \mathrm{~mm}$ respectively from the camera. The camera parameters for the capture were $f=18 \mathrm{~mm}$, and to allow the 3D scene to be captured sharply we used a large $\mathrm{f}$ number, $f / 22$.

The next step is to calculate, from the captured Els, the exceeded-in-parallax microimages. To this end we used the SPOC

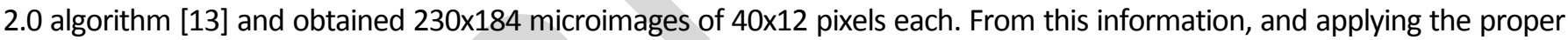
cropping corresponding to any possible lateral position of the observer, we calculated 28 integral frames composed each by $12 \times 12$ cropped microimages. Finally, in order to fully adapt the frames to the integral monitor we resized them so that the microimages had $11.7 \times 11.7$ pixels each. Additionally we calculated other set of 28 integral frames corresponding to a second position of the reference plane.

For the eye-tracking system we used a Kinect v2 device centered laterally with the integral monitor. Kinect v2 works with IR time of flight technology, which enables it to provide high-accuracy depth maps [27-29]. In addition, we used a Microsoft SDK that allows the Kinect to track the position of moving observers. The tracking method works in a two-step process. First, it finds the observer's head position and, second, the eye-pupils positions. Due to using IR technology, the Kinect can operate in low light conditions and, therefore, track the observer position even in dark rooms. In order to allow the observer to be tracked, she/he needs to move inside the field of view and the depth tracking range of the Kinect. The Kinect viewing angles are $70^{\circ}(\mathrm{H}) \times 60^{\circ}(\mathrm{V})$. Although the depth tracking range covers from $500 \mathrm{~mm}$ to $8000 \mathrm{~mm}$, the range in which the depth map is provided with optimal accuracy is reduced to $1000-4500 \mathrm{~mm}$. In this optimal range, the accuracy for the determination of the lateral position of a given object (like the eye pupil) is of $0.137^{\circ}$. For the depth distance the accuracy is of $2 \mathrm{~mm}$ [29]. Some characteristics of Kinect v2 are illustrated in

Fig. 6. 


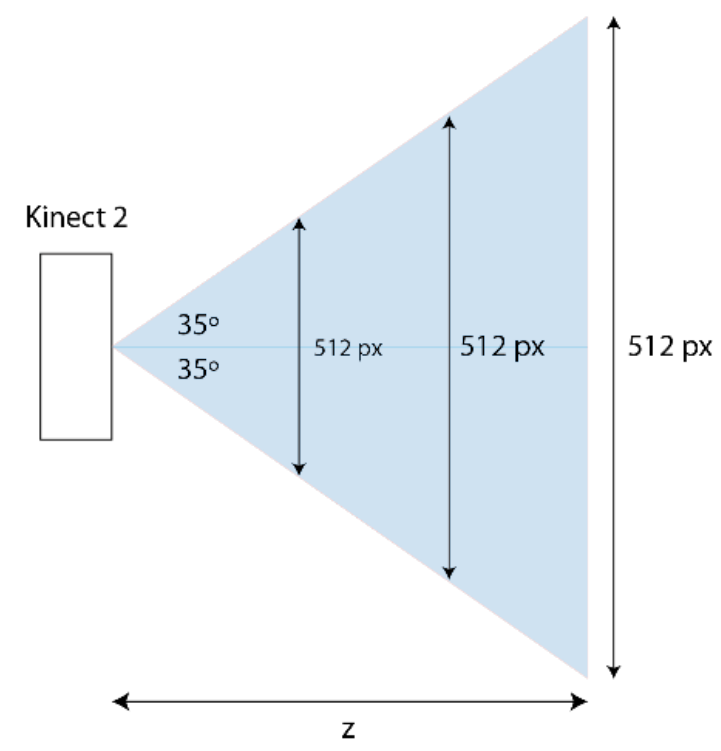

Fig. 6.- Kinect v2 tracking area. The number of pixels of the IR sensor is 512 and limits the lateral tracking resolution.

In order to know the correspondence between the pixels of the IR sensor and the sectors in front of the integral monitor, we need to translate from sensor pixels unit to $\mathrm{mm}$. The lateral tracking resolution in $\mathrm{mm}$ depends on the distance from the Kinect $\mathrm{v} 2$ to the object being tracked. It can be calculated as:

$$
\begin{gathered}
T A=2 Z \tan \left(35^{\circ}\right) \\
I R_{m m}=\frac{T A}{I R_{p i x}}
\end{gathered}
$$

where $T A$ (tracking area) is the Kinect v2 field of view in millimeters, $I R_{\text {pix }}$ is the number of pixels of the $I R$ sensor, $I R_{\mathrm{mm}}$ is the size of an IR pixel at the observer position, and $z$ is the distance from the integral monitor to the observer. Then the size, $S$, of each sector as expressed in units of Kinect IR sensor pixels, is

$$
S=\frac{\Delta(z)}{I R_{m m}}
$$

Using Eq. (3), (4) and (5) brings an interesting result: the size of each sector in IR pixel unit is 6 pixels and is independent of the observer distance to the integral monitor. Therefore, we can divide the IR sensor in sets of 6 pixels and assign to each set of pixels its corresponding pre-generated image.

To carry out the experiment, the control software selects the integral frame to be displayed depending on the 3D position of the observer. When the Kinect v2 communicates to the software that the observer has moved from a set of 6 pixels to a different set of 6 pixels, the control software changes the displayed integral frame to the next one. In addition, the software will also change the displayed image to another one with a different reference plane depending on the observer's depth position. As we have prepared images with two different reference planes, we have defined two arbitrary depth planes in the 3D space in front of the integral monitor. The first plane is at $z=1940 \mathrm{~mm}$ and the second one is at $z=2040 \mathrm{~mm}$. The reference plane of the displayed image will depend on which depth plane the observer is situated. Note that the reason we choose a $100 \mathrm{~mm}$ range to trigger the change is because a displacement in depth position of that quantity is enough to provide a good visualization experience to the observer.

To demonstrate the utility of our method we have recorded 5 videos. In Media 1 and Media 2 we compare our proposal with an integral monitor operating in conventional mode. In both cases, the observer moves laterally in front of the integral monitor at $z=2040 \mathrm{~mm}$ from it. For this value of $z$ the size of the viewing area is $V A=16 \mathrm{~mm}$. Therefore, the restriction 
over the lateral position of the observer is very tight if the integral monitor is working in the conventional mode. Also for the conventional mode the viewing angle is $\beta=0.3^{\circ}$. In Media 1 we use a conventional integral monitor configuration, without tracking and always displaying the same image. On the other hand, Media 2 corresponds to the proposed technique where the eye-tracking is enabled and the displayed integral frame is adapted to the observer position. Taking into account the size of the uncropped microimages (40x12 pixels) the viewing angle rises to $\beta=14.8^{\circ}$. Thus, the observer can change her/his position laterally along an interval larger than $1.000 \mathrm{~mm}$.

To record the videos, we used a digital camera mounted on a moving platform. In both experiments the lateral displacement was approximately $d=800 \mathrm{~mm}$. From the videos it is apparent, first, that our technology permits the observer to make large displacements in parallel to the screen without perceiving any flipping or crosstalk. Additionally the parallax has been enlarged substantially (note that in Media 1 the observer is perceiving repeatedly the same perspectives). In Fig. 7 we show four frames of Media 1 and Media 2.

Media 3 shows the experimental results for an observer moving towards and backwards the integral monitor. Again, for this video the digital camera simulates the observer. Specifically, when the observer is far from the monitor, the reference plane is set at the front of the (blue and yellow) container, whereas the rest of the scene is reconstructed behind the integral monitor. As usually the best quality of reconstruction is obtained at the reference plane, and therefore other parts of the 3D scene (like the house) appear slightly blurred. Later in the video, when the observer gets closer the monitor the reference plane is set at the house facade. In this case the rest of the 3D scene is reconstructed in front of the monitor, and the container is perceived with some blurring. The magnifying effect that can be perceived is due to the fact that when one approaches to one scene it is perceived with bigger angular size.

In Media 4 we show how the integral monitor adapts its image, in real time, to the lateral movement of a human observer. Note that the video is not recorded from the observer point of view, but from other perspective. Thus, what we are showing is not the image as perceived by the observer (shown in Media 2). Analogously, in Media 5 we show how the integral monitor adapts the displayed image to the changes in depth position of the human observer. Again, this video is showing a perspective different from the one perceived by the observer (which is shown in Media 3). Thus, the strong crosstalk shown is perceived by the camera used for recording the video, but not by the observer. In Fig. 8 we show a single frame extracted from Media 5. 

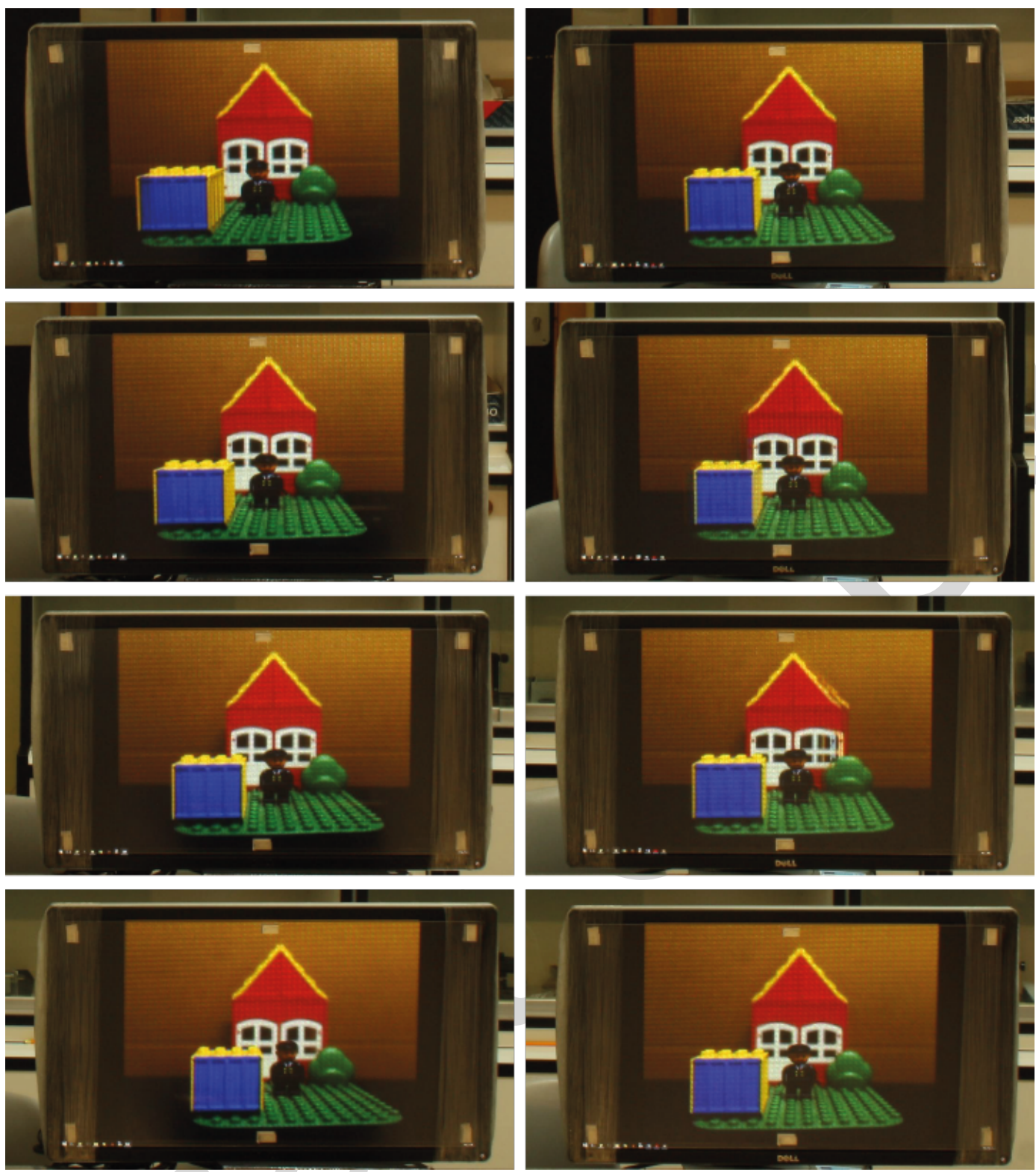
Fig. 7.- Comparison between our method (left column) with a conventional integral monitor configuration (right column). The images were captured in different lateral positions in front of the integral monitor. The total displacement was approximately $800 \mathrm{~mm}$. On the conventional Inl configuration, the crosstalk can be seen at the left side of the house (second image) and on the right side of the house (third image).

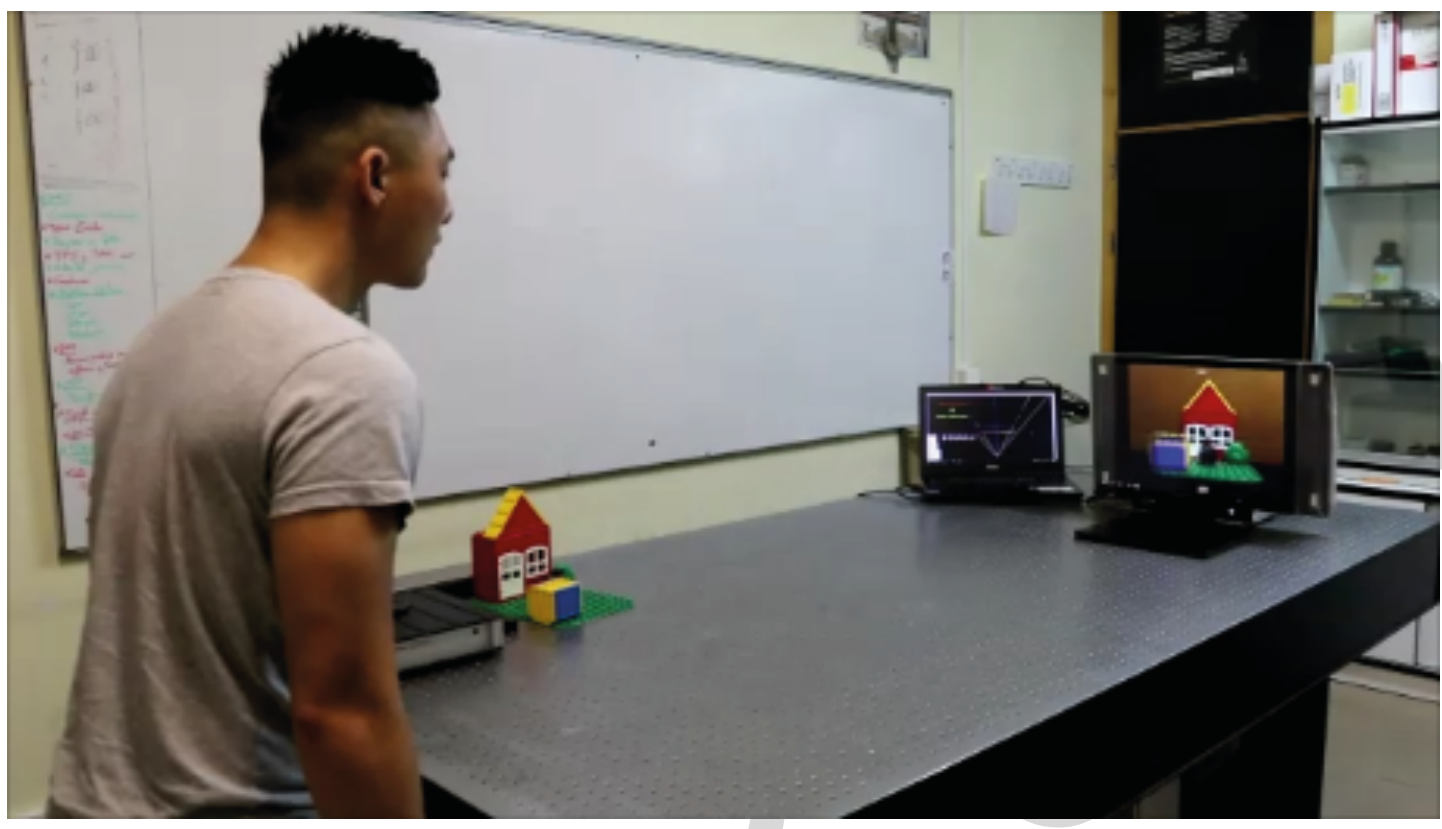

Fig. 8.- The display system is composed by an integral monitor, a Kinect v2 device, and a computer that receives the information from the Kinect and transmit to the monitor the corresponding integral frame. The image displayed on the integral monitor is adapted to the 3D position of the observer in real time.

\section{CONCLUSION}

We have proposed a method to improve the viewing angle and the parallax provided by integral monitors, and therefore the observer experience. The method uses an eye-tracking system based in Kinect technology. This technology can work in low luminance environment, and the observers do not need to wear any special glasses or IR clip. But the main advance of our method is that it can work with real 3D scenes that are composed by many depth sections and can have a bright background. The technique is based in the smart cropping of microimages that have excess parallax. An additional advantage of our method is that the reference plane can be selected at will. Through our experiments we have demonstrated, at least for a static image, that it is possible to adapt in real time the displayed 3D image to the observer location.

Funding Information. This work was supported in part by the Ministerio de Economía y Competitividad, Spain [DPI 201566458-C2-1-R], and by Generalitat Valenciana, Spain [project PROMETEOII/2014/072]. A. Dorado acknowledges a predoctoral fellowship from the MINECO [BES-2013-064867]. S. Hong acknowledges a predoctoral grant from University of Valencia [UV-INVPREDOC15-265754]. B. Javidi acknowledges support under [NSF/IIS-1422179].

\section{References}

1. G. Lippmann, “Epreuves reversibles donnant la sensation du relief," J. Phys. Theor. Appl. 7, 821-825 (1908).

2. E. H. Adelson and J. Y. A. Wang, "Single lens stereo with plenoptic camera," IEEE Trans. Pattern Anal. Mach. Intell. 14, 99-106 (1992).

3. J.-H. Park, K. Hong, and B. Lee, "Recent progress in three-dimensional information processing based on integral imaging," Appl. Opt. 48, H77-H94 (2009) .

4. H. Navarro, G. Saavedra, A. Molina, M. Martinez-Corral, R. Martinez-Cuenca, and B. Javidi "Optical slicing of large scenes by synthetic aperture integral imaging" Proc. SPIE 7690, 7690-0M (2010). 
5. X. Xiao, B. Javidi, M. Martinez-Corral, and A. Stern, "Advances in Three-Dimensional Integral Imaging: Sensing, Display, and Applications" Appl. Opt. 52, 546-560 (2013)

6. H. Navarro, A. Dorado, G. Saavedra, and M. Martinez-Corral, "Three-Dimensional Imaging and Display through Integral Photography" J. Inf. Commun. Converg. Eng. 12, 89-96 (2014).

7. M. Martinez-Corral, A. Dorado, J. C. Barreiro, G. Saavedra, and B. Javidi, "Recent advances in the capture and display of macroscopic and microscopic 3D scenes by integral imaging", Proc. IEEE 105, 825-836 (2017).

8. G. Park, J. Hong, Y. Kim, and B. Lee, "Enhancement of Viewing Angle and Viewing Distance in Integral Imaging by Head Tracking," in Advances in Imaging, OSA Technical Digest (CD) (Optical Society of America, 2009), paper DWB27.

9. G. Park, J.-H. Jung, K. Hong, Y. Kim, Y.-H. Kim, S.-W. Min, and B. Lee, "Multi-viewer tracking integral imaging system and its viewing zone analysis," Opt. Express 17, 17895-17908 (2009).

10. S. Hong, D. Shin, J.-J. Lee, and B.-G. Lee, "Viewing Angle-Improved 3D Integral Imaging Display with Eye Tracking Sensor", J. Inf. Commun. Converg. Eng. 12, 208-214 (2014).

11. X. Shen, M. Martinez-Corral, and B. Javidi, "Head Tracking three-dimensional integral imaging display using smart pseudoscopic-to orthoscopic conversion", J. Display Technol. 12, 542-548 (2016).

12. Z-L. Xiong, Q.-H. Wang, S.-L. Li, H. Deng, and C.-C. Ji, "Partially-overlapped viewing zone based integral imaging system with super wide viewing angle," Opt. Express 22, 22268-22277 (2014)

13. M. Martinez-Corral, A. Dorado, H. Navarro, G. Saavedra, and B. Javidi, "Three-dimensional display by smart pseudoscopic-to-orthoscopic conversion with tunable focus," Appl. Opt. 53, 19-25 (2014).

14. H. Hiura, T. Mishina, J. Arai, K. Hisatomi, Y. Iwadate, T. Ito, and S. Yano, "A study on accommodation response and depth perception in viewing integral photography," Proceedings of 3D Systems and Applications, Osaka (Japan) (2013), paper P2-2.

15. H. Hiura, K. Komine, J. Arai, and T. Mishina, "Measurement of static convergence and accommodation responses to images of integral photography and binocular stereoscopy," Opt. Express 25, 3454-3468 (2017).

16. R. Ng, M. Levoy, M. Brédif, G. Duval, M. Horowitz, and P. Hanrahan, "Light field photography with a handheld plenoptic camera," Tech. Rep. CSTR. 2 (2005).

17. R. Ng, "Digital light field photography," Ph.D. dissertation, (Stanford University, 2006).

18. H. Navarro, J. C. Barreiro, G. Saavedra, M. Martinez-Corral, and B. Javidi, "High-resolution far-field integralimaging camera by double snapshot," Opt. Express 20, 890-895 (2012).

19. T. Iwane, "Light field camera and IP display," Proceedings of 3D Systems and Applications, Osaka (Japan) (2013), p. 32.

20. A. Dorado, G. Saavedra, J. Sola-Pikabea, and M. Martinez-Corral, "Integral imaging monitors with an enlarged viewing angle" J. Inf. Commun. Converg. Eng. 13, 132-138 (2015).

21. A. Dorado, M. Martinez-Corral, G. Saavedra, and S. Hong, "Computation and display of 3D movie from a single integral photography", J. Display Technol. 12, 695-700 (2016).

22. S. Hong, G. Saavedra, and M. Martinez-Corral, "Full parallax 3D display from Kinect v1 and v2", Opt. Eng. 56, 41305 (2016).

23. R. Martinez-Cuenca, G. Saavedra, A. Pons, B. Javidi and M. Martinez-Corral, "Facet braiding: a fundamental problem in Integral Imaging", Optics Letters 32, 1078-1080 (2007)

24. H. Navarro, R. Martinez-Cuenca, A. Molina-Martín, M. Martinez-Corral, G. Saavedra, and B. Javidi, "Method to remedy image degradations due to facet braiding in 3D integral imaging monitors", J. Disp. Technol. 6, 404-411 (2010)

25. Y. Kim, G. Park, J.-H. Jung, J. Kim, and B. Lee, "Color moiré pattern simulation and analysis in three-dimensional integral imaging for finding the moiré-reduced tilted angle of a lens array," Appl. Opt. 48, 2178-2187 (2009).

26. J. S. Jang and B. Javidi, "Three-dimensional synthetic aperture integral imaging," Opt. Lett. 27, 1144-1146 (2002).

27. "Kinect for Xbox One Components and Specifications", http://dev.windows.com/en-us/kinect/hardware (2013).

28. R. Smeenk, "Kinect v1 and Kinect v2 fields of view compared," http://smeenk.com/kinect-field-of-viewcomparison. (2014) 
29. D. Pagliari and L. Pinto, "Calibration of Kinect for Xbox one and comparison between the two generations of Microsoft sensors," Sensors 15, 27569- 27589 (2015).

Adrian Dorado was born in Spain in 1988. He received the B.Sc. and M.Sc. degrees in physics from the University of Valencia, Spain, in 2011 and 2012, respectively. Since 2010, he has been with the 3D Imaging and Display Laboratory, Optics Department, University of Valencia. His research interests include 3D imaging acquisition and display.

Seokmin Hong received the B.Eng. and M.Sc. degrees in digital and visual contents from Dongseo University, Busan, South Korea, in 2012 and 2014, respectively. In 2012, Dongseo University honored him with the B.Eng. Extraordinary Award. Since 2015, he has been working with the 3D Imaging and Display Laboratory, Optics Department, University of Valencia, Spain. His research interests are image processing, computer vision, and applied computer science.

Genaro Saavedra received the B.Sc. and Ph.D. degrees in physics from Universitat de València, Spain, in 1990 and 1996, respectively. His Ph. D. work was honored with the Ph.D. Extraordinary Award. He is currently Full Professor with Universitat de València, Spain. Since 1999, he has been working with the "3D Display and Imaging Laboratory", at the Optics Department. His current research interests are optical diffraction, integral imaging, 3D high-resolution optical microscopy and phase-space representation of scalar optical fields. He has published on these topics about 50 technical articles in major journals and 3 chapters in scientific books. He has published over 50 conference proceedings, including 10 invited presentations.

Bahram Javidi (Fellow, IEEE) received the B.S. degree from George Washington University, USA, and the M.S. and Ph.D. degrees from the Pennsylvania State University, USA, all in electrical engineering. He is the Board of Trustees Distinguished Professor at the University of Connecticut, Storrs, CT, USA. He has over 1000 publications, including nearly 450 peer reviewed journal articles, over 450 conference proceedings, including over 120 Plenary Addresses, Keynote Addresses, and invited conference papers. His papers have been cited 34000 times according to the Google Scholar Citations (h-index $=87$, i10-index $=537$ ). He is a co-author on nine Best Paper Awards. Prof. Javidi received the Quantum Electronics and Optics Prize for Applied Aspects by the European Physical Society in June 2015. He has been named Fellow of several scientific societies, including IEEE, OSA, and SPIE. In 2010, he was the recipient of the George Washington University's Distinguished Alumni Scholar Award, University's highest honor for its alumni in all disciplines. In 2008, he received a Fellow award by John Simon Guggenheim Foundation. He received the 2008 IEEE Donald G. Fink prized paper award among all (over 150) IEEE Transactions, Journals, and Magazines. In 2007, The Alexander von Humboldt Foundation awarded him with the Humboldt Prize for outstanding U.S. scientists. He received the Technology Achievement Award from the SPIE in 2008. In 2005, he received the Dennis Gabor Award in Diffractive Wave Technologies from SPIE. He was the recipient of the IEEE Photonics Distinguished Lecturer Award twice in 2003/2004 and 2004/2005. He was awarded the IEEE Best Journal Paper Award from IEEE TRANSACTIONS ON VEHICULAR TECHNOLOGY twice in 2002 and 2005. Early in his career, the National Science Foundation named him a Presidential Young Investigator and he received The Engineering Foundation and the IEEE Faculty Initiation Award. He was selected in 2003 as one of the nation's top 160 engineers between the ages of 30 to 45 by the National Academy of Engineering (NAE) to be an invited speaker at The Frontiers of Engineering Conference which was co-sponsored by The Alexander von Humboldt Foundation. He is an alumnus of the Frontiers of Engineering of The National Academy of Engineering since 2003. $\mathrm{He}$ has served on the Editorial Board of the Proceedings of the IEEE Journal [ranked \#1 among all electrical engineering journals], the advisory board of the IEEE PHOTONICS JOURNAL, and he was on the founding board of editors of IEEE/OSA Journal of Display Technology.

Manuel Martinez-Corral received the Ph. D. degree in Physics (Best Thesis Award) from the University of Valencia in 1993. Currently Full Professor of Optics at the University of Valencia, he co-leads the "3D Imaging and Display Laboratory". He was elected Fellow of the SPIE and Fellow of the OSA in 2010 and 2017, respectively. His research interest includes resolution procedures in 3D scanning microscopy, and 3D imaging and display technologies. He 
has supervised on these topics $12 \mathrm{Ph}$. D. theses (three honored with the Best Thesis Award), published over eighty technical articles in major journals (which received about 2.300 citations), and pronounced a number of invited and keynote presentations in international meetings. He is co-chair of the Three-Dimensional Imaging, Visualization, and Display Conference within the SPIE meeting in Defense, Security, and Sensing, and Topical Editor of the OSA Applied Optics.

13 